\title{
A GUI for Automatic Extraction of Signature from Image Document
}

\author{
Chandra Mohan Gautam \\ School of IT \\ RGPV BHOPAL(M.P)
}

\author{
Sanjeev Sharma \\ School of IT \\ RGPV BHOPAL(M.P)
}

\author{
Jitendra Singh Verma \\ School of IT \\ RGPV BHOPAL(M.P)
}

\begin{abstract}
A New method for Extracting signature from image document is proposed based on the auto cropping method. This method improves the performance of security system based on signature images as well as provides the region of interest of the used image for the biometric system. This method also reduces the time cost associated with signature detection. Region of Interest in an image document is taking attention in research area. In this method, the signature document image converted into the binary image. This Binary image is normalized (resize) the signature image. To show the signature effective areas in signature image resizing may be required. Now we are performing Adaptive thresholding because it dynamically set all pixels whose intensity values are above a threshold to a foreground value and all the remaining pixels to a background value over the signature document image. This signature document image has some discontinuity between the pixels to remove this discontinuity we are using morphology. This morphological method uses bridge to connect the pixels and remove operator to remove the interior pixel region. The remaining pixel makes the signature image skeleton. This skeleton is used to select the signature Region of Interest (ROI) using auto cropping method. Auto cropping is the fast procedure to select the ROI. In this auto cropping method we are using Image Station Automatic Elevations (ISAE) technique to select the connected pixel which sizes are greater than 250 pixels. This cropped signature has no garbage region it crops only the ROI of signature image. This method takes less processing time then other methods. To extract the features of cropped image we are using the 'Sobel' edge detection to identify the points in a digital image at which the image brightness changes sharply or, more formally has discontinuities.
\end{abstract}

\section{Keywords: Binarization, ROI, ISAE, Adaptive}

Thresholding, Image normalization (resizing), Morphology, Auto cropping.

\section{INTRODUCTION}

Biometric image preprocessing is interesting field in Digital image processing research area. This field has little work done. Biometric images are used for biometric authentication and bio crypto key generation. Signature is one of behavioral biometric types. Signatures are a special case of handwriting subject to intra personal variation and interpersonal differences. This variability makes necessary to analyze signatures as complete images and not as collection of letters and words [1]. Any signature verification system built on five stages: data acquisition, pre-processing, feature extraction, comparison process and performance evaluation [2]. We concern in this work on the pre-processing stage, where this stage consists of several steps: one of these steps is noise removing [3]. Another one which proposed in [4] is a noise filtering which is applied to remove the noise caused by the scanner, where the image is cropped to the bounding rectangle of the signature, then a transformation from color to grayscale and so forth to black and white, finally the bounding pixels of the signature are marked and removed without eliminating the ending point until get the signature skeleton, i.e. Skeletonization, unless a pixel has less than three neighbors left, or any path between the neighbor pixels would be interrupted by the action. A non uniformity correction for sensor elements, localization of the signature in the picture, extraction of the signature from the background, slicing, thresholding segmentation, normalization and data reduction[5] are another steps for pre-processing. An experimental database has been acquired on a restricted size grid [6], Normalization includes basic techniques like, scaling, translation, and rotation etc [5] presented to avoid signature rotational problems. A given signature images threshold using Adaptive Thresholding Method [10]. A proposed approach consists of: image binarization, image normalization (resize), image thresholding, image enhancement using morphological operators, edge detection, and finally cropping. In signature verification technology, many algorithms used manual cropping such as in [2], while automatic cropping algorithms suffers from cropping information lost, since it is drawing rectangle rounded the signature then transfer the region inside it into another matrix (false rectangle). The region of interest term referred to object interest sub region of an image, leaving other regions unchanged. In signature verification system the signature itself is the ROI.

\section{RELATED WORK}

1. Jason Forshaw, Ryan Volz [7] in this work author, identifying the region of interest in a breast MRI image is a tedious manual process that would be much more suited for a computer, but it is necessary for performing useful postprocessing on the image for breast cancer research and treatment. We devise such an approach following the concept that an MRI image can be sectioned into a number of contiguous regions based on pixel intensity clusters and edge detection. We then train an SVM algorithm to identify the specific sections that belong in the region of interest. Although our approach is not yet ready to replace hand-drawn regions of interest, the technique shows promise for eventually being able to do so.

2. Shih-Yin Ooi, Andrew Beng-Jin Teoh, Thian-Song Ong [8] in this work author says the offline signature verification rests on the hypothesis that each writer has similarity among signature samples, with small distortion and scale variability. In this paper we propose a novel method to increase the accuracy in biometric matching which we term biometric strengthening. We reported $1.1 \%$ equal error rate (EER) over the independent database on random forgery, while casual 
forgery on EER $1.2 \%$ and lastly skilled forgery on EER $2.1 \%$ along the paper. Our experiments show that biometric strengthening reduces the false acceptance rate (FAR) and false rejection rate (FRR) by increasing the disparity between the features of the two persons, which tends to tolerate more intrapersonal variance which can reduce the FRR without increasing the probability of false, accepts.

3. Miguel A. Ferrer, Jesu's B. Alonso, Carlos M. Travieso [9] in this work author presents a set of geometric signature features for offline automatic signature verification based on the description of the signature envelope and the interior stroke distribution in polar and Cartesian coordinates. The features have been calculated using 16 bits fixed-point arithmetic and tested with different classifiers, such as hidden Markov models, support vector machines, and Euclidean distance classifier. The experiments have shown promising results in the task of discriminating random and simple forgeries

4. Derek Bradley, Gerhard Roth [10] in this work author told us Image thresholding is a common task in many computer vision and graphics applications. The goal of thresholding an image is to classify pixels as either "dark" or "light". Adaptive thresholding is a form of thresholding that takes into account spatial variations in illumination. We present a technique for real-time adaptive thresholding using the integral image of the input. Our technique is an extension of a previous method. However, our solution is more robust to illumination changes in the image. Additionally, our method is simple and easy to implement. Our technique is suitable for processing live video streams at a real-time frame-rate, making it a valuable tool for interactive applications such as augmented reality.

5. MamtaJuneja, Parvinder Singh Sandhu [11] in this work author says, Edges characterize boundaries and are therefore considered for prime importance in image processing. Edge detection filters out useless data, noise and frequencies while preserving the important structural properties in an image. Since edge detection is in the forefront of image processing for object detection, it is crucial to have a good understanding of edge detection methods. In this paper the comparative analysis of various Image Edge Detection methods is presented. The evidence for the best detector type is judged by studying the edge maps relative to each other through statistical evaluation. Upon this evaluation, an edge detection method can be employed to characterize edges to represent the image for further analysis and implementation. It has been shown that the 'sobel' edge detection algorithm performs better than all these operators under almost all scenarios.

6.Vamsi Krishna Madasu, Mohd. HafizuddinMohd. Yusof, M. Hanmandlu[16] in author says in the Automatic Extraction of Signatures from Bank Cheques and other Documents. An innovative approach for extracting signatures from bank cheque images and other documents is proposed based on the integration of the crop method with the sliding window technique. The idea is to estimate the approximate area in which the signature lies using the sliding window technique. In this approach, a window of adaptable height and width is moved over the image; one pixel at a time and the density of pixels within the window is calculated. This density is then used to find the entropy, which in turn helps fit the box that can segment the signature. The signatures thus extracted are then fed to a known fuzzy based off-line signature verification and forgery detection system. The proposed method has been applied with almost $100 \%$ success on several bank cheques from India, Malaysia and Australia. Signature extraction has also been shown on two typical types of documents which have varied and noisy backgrounds.

\section{PROPOSED METHODOLOGY}

A New method for Extracting signature from image on document is proposed base the auto cropping method. This method improve the performance of security system based on signature image as possible the region of interest of the used image for the biometric system. This method also reduces the time cost associated with signature. Region of Interest in an image document is taking attention in research area.

To extract the signature Region of Interest (ROI) using auto cropping method from document image. Auto cropping is the fast procedure to extract the ROI. In this auto cropping method we are using image segmentation and extraction. In this auto cropping method we are using Image Station Automatic Elevations (ISAE) technique to select the connected pixel which sizes are greater than 250 pixels. This cropped signature has no garbage region it crops only the ROI of signature image. This signature can take less processing time then the original signature image

\subsection{METHOD}

The main steps of the method are given below:

STEP -1.Pre- processing

Read the input Image $\mathrm{I}(\mathrm{x}, \mathrm{y})$ Document from image Database.

STEP -2. Applying Binarization method, to convert original image into binary image and apply a liner filter method.

STEP -3. Applying Normalization to resize the image and fix the image area.

STEP -4.Applying Adaptive Thresholding to set all pixel of image document dynamically.

STEP -5. Applying morphology technique, to connect the pixels and remove operator to remove the interior pixel region.

STEP -6. Applying Image Segmentation and cropping ISAE method to extract the signature from an Image document.

STEP -7.Applying 'Sobel' Edge Detection to extract the features of cropped images.

STEP -8 finally obtains the extracted Signature image as show in fig. 1.

\subsection{MODEL ARCHITECTURE}

The Block Diagram of the extract signature from image document method is show in the fig. 1 . 


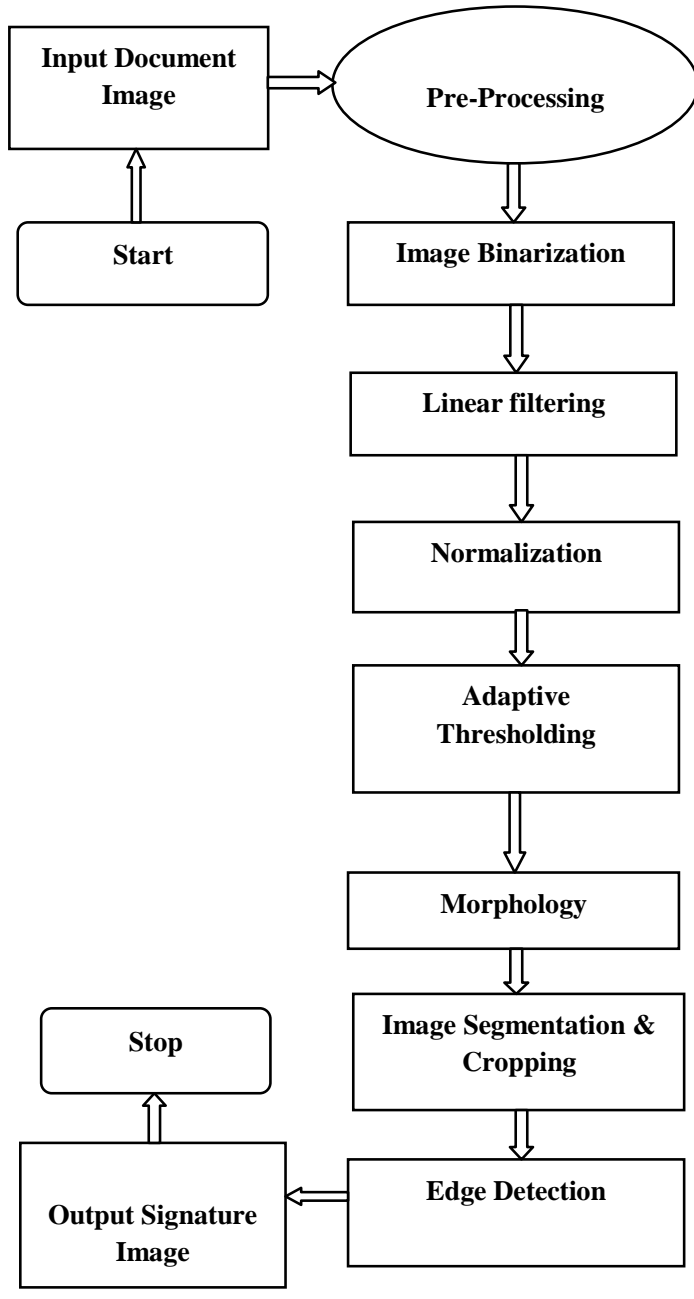

Fig. 1 Block Diagram of Proposed Method

\subsection{MODEL DESCRIPTION}

The above shown model is a new model to extract signature from image document. The model used the properties of the MATLAB SIMULINK model system. It is introduced for the segmentation\& cropping the signature from image document file. The model contains more important blocks and result display blocks

\section{Binarization}

This Binary image format also stores an image as a matrix but can only color a pixel black or white (and nothing in between). It assigns a 0 for black and a 1 for white. 0 and 1 values are showing the weight of the pixel, as show in fig. 2 .

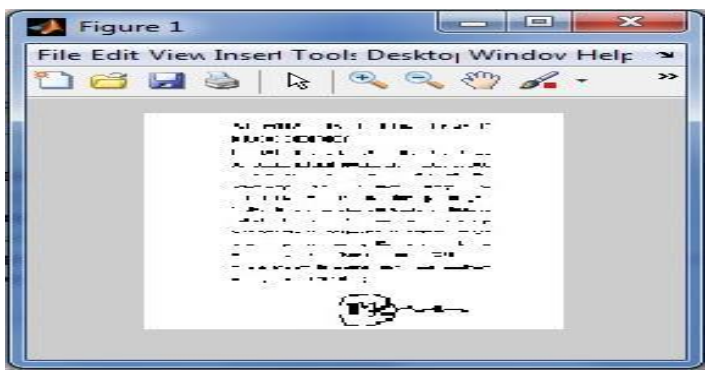

Fig. 2 Binarization
The first step in given approach is to convert the document signature into binary form, i.e. black and white pixels. Working in this form is more useful than any other form, since it is easy to work with 2 bits representation of image.

The required time to process colored image is longer than binary one, as example applying radon transform on different types of image (colored Vs binary) shows in fig.3 the following deference

\section{Colored image (2D) $\quad$ Binary image}

\begin{tabular}{|c|c|c|c|c|c|c|c|c|}
\hline & 296 & 297 & 298 & $i$ & & 296 & 297 & 298 \\
\hline 26 & 240 & 253 & 255 & 26 & 1 & 1 & 1 & 1 \\
\hline 27 & 255 & 255 & 255 & 27 & 1 & 1 & 1 & 1 \\
\hline 28 & 249 & 252 & 252 & 28 & 1 & 1 & 1 & 1 \\
\hline 29 & 250 & 255 & 253 & 29 & 1 & 1 & 1 & 1 \\
\hline 30 & 255 & 254 & 253 & 30 & 1 & 1 & 1 & 1 \\
\hline 31 & 249 & 250 & 252 & 31 & 1 & 1 & 1 & 1 \\
\hline 32 & 255 & 252 & 254 & 32 & 1 & 1 & 1 & 1 \\
\hline 33 & 255 & 253 & 254 & 33 & 1 & 1 & 1 & 1 \\
\hline 34 & 249 & 253 & 254 & 34 & 1 & 1 & 1 & 1 \\
\hline
\end{tabular}

Fig.3 Colored Vs Binary Image Matrixes

\section{Image normalization (resizing)}

Normalization is one of the most important steps of image pre-processing techniques. Experimental Database has been acquired on a restricted size grid; Normalization includes basic techniques like, scaling, translation, and rotation etc. People normally scale their signatures to fit the area available for the signature. However size difference may be a problem in comparing the two signatures. Therefore signatures are normalized with respect to width, height or both as show in fig. 4.

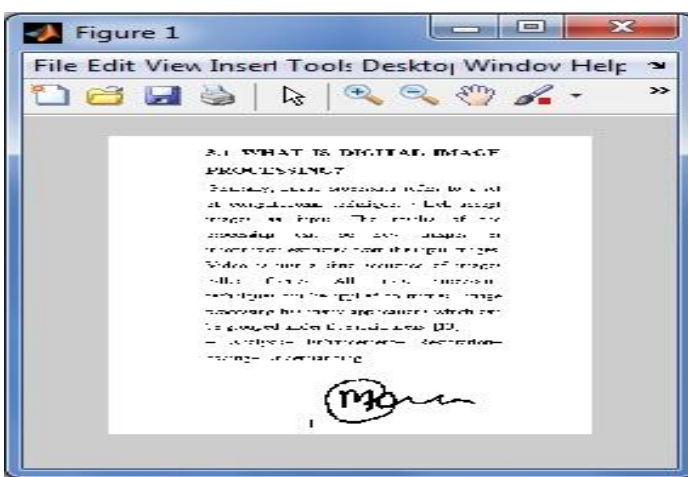

Fig.4 Image normalization (resizing)

In this work, the Binary image is normalized (resize) the signature image. To show the signature effective areas in signature image resizing may be required.

\section{Linear filter}

Linear filters in the time domain process time-varying input signals to produce output signals, subject to the constraint of linearity. These results from systems composed solely of components (or digital method) classified as having a linear response. Most filters implemented in analog electronics, in digital signal processing, or in mechanical systems are classified as causal, time invariant, and linear. However the general concept of linear filtering is broader, also used in statistics, data analysis This includes non causal filters and filters in more than one dimension such as would be used in image processing; those filters are subject to different constraints leading to different design methods, which are 
discussed Low pass filtering, otherwise known as "smoothing", is employed to remove high spatial frequency noise from a digital image. Noise is often introduced during the analog-to-digital conversion process as a side-effect of the physical conversion of patterns of light energy into electrical patterns

There are several common approaches to removing this noise:

- If several copies of an image have been obtained from the source, some static image, then it may be possible to sum the values for each pixel from each image and compute an average. This is not possible, however, if the image is from a moving source or there are other time or size restrictions.

- If such averaging is not possible, or if it is insufficient, some form of low pass spatial filtering may be required. There are two main types:

a. Reconstruction filtering, where an image is restored based on some knowledge of the type of degradation it has undergone. Filters that do this are often called "optimal filters".

b. Enhancement filtering, which attempts to improve the (subjectively measured) quality of an image for human or machine interpretability. Enhancement filters are generally heuristic and problem oriented.

\section{Thresholding}

Thresholding is used to segment an image by setting all pixels whose intensity values are above a threshold to a foreground value and all the remaining pixels to a background value. There are many methods are used for thresholding. In this step we are using Adaptive Thresholding. Adaptive thresholding typically takes a grayscale or color image as input and, in the simplest implementation, outputs a binary image representing the segmentation. For each pixel in the image, a threshold has to be calculated. If the pixel value is below the threshold it is set to the background value, otherwise it assumes the foreground value as show in fig 5 .

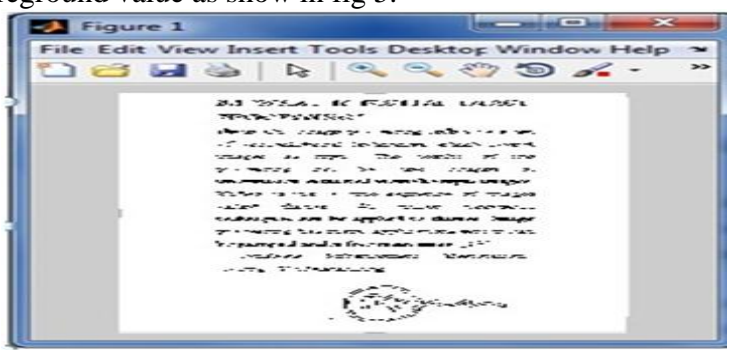

Fig.5 Adaptive Thresholding

There are two main approaches to finding the threshold: (i) the Chow and Kaneko approach and (ii) local thresholding. The assumption behind both methods is that smaller image regions are more likely to have approximately uniform illumination, thus being more suitable for thresholding. Chow and Kaneko divide an image into an array of overlapping sub images and then find the optimum threshold for each sub image by investigating its histogram. The threshold for each single pixel is found by interpolating the results of the sub images. The drawback of this method is that it is computational expensive and, therefore, is not appropriate for real-time applications. An alternative approach to finding the local threshold is to statistically examine the intensity values of the local neighborhood of each pixel. The statistic which is most appropriate depends largely on the input image. Simple and fast functions include the mean of the local intensity distribution.

\section{$\mathrm{T}=$ mean}

The median value,

$$
\mathrm{T}=\text { median }
$$

or the mean of the minimum and maximum value

$$
T=\frac{\operatorname{Max}+\operatorname{Min}}{2}
$$

The size of the neighborhood has to be large enough to cover sufficient foreground and background pixels, otherwise a poor threshold is chosen. On the other hand, choosing regions which are too large can violate the assumption of approximately uniform illumination. This method is less computationally intensive than the Chow and Kaneko approach and produces good results for some applications

\section{Morphology}

Morphology is a technique to creating an image noise free. Morphology usually divided into binary mathematical morphology which operates on binary images and gray level images. The Mathematical Morphology is calculated based on simple mathematical concepts from set theory, morphological operators are useful for binary image, it views binary image as asset of its foreground (1-valued) pixels, and set operations such as union and intersection can be applied directly to binary image sets [15]. In our approach, we used bridge to connect discontinuity of pixels, then used remove operator to remove interior pixels keeping the boundaries of signature. A skeletonization is used to remove pixels on the boundaries of objects but does not allow objects to break apart. The pixels remaining make up the image skeleton as show in fig. 6 .

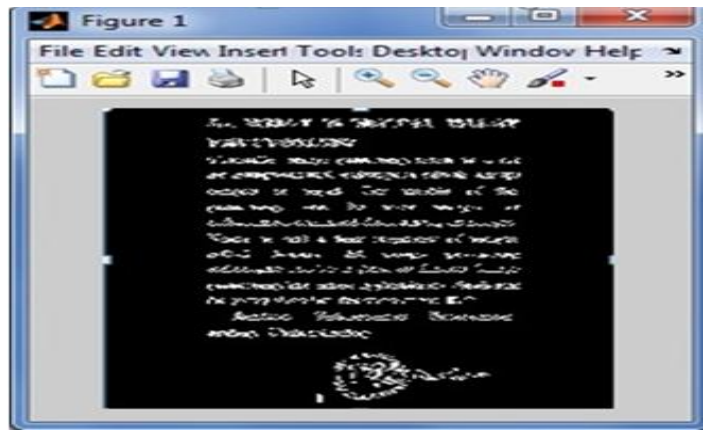

Fig.6 Morphology

We did not use thinning because of this operator may cause lost of information, but using remove and bridge operator save the greatest amount of information, since they keep the boundary of signature.

\section{Image Segmentation and Cropping}

The objective of segmentation is to partition an image into regions. We can find the region based on the point detection, line detection, and edge detection by finding boundaries between regions based on discontinuities in gray levels and via thresholds based on the distribution of pixel properties 
such as gray level values or color. Here the segmentation techniques that are based on finding the regions directly [12].As show in fig. 7.

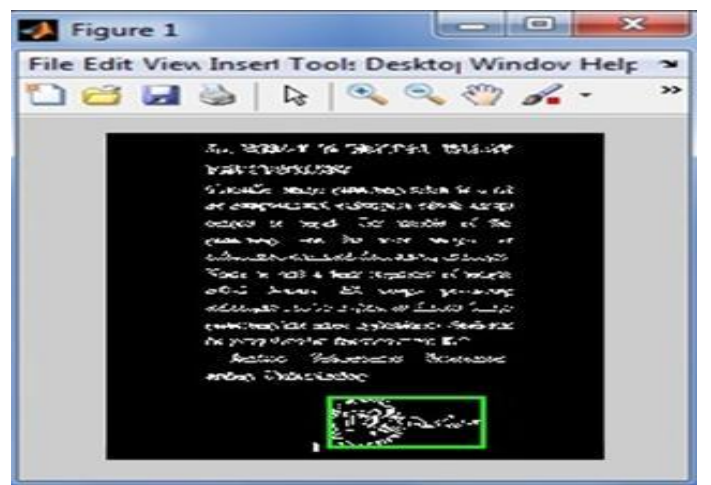

Fig.7 Region of Interest

\section{Image Station Automatic Elevations (ISAE) Segmentation method}

Image Station Automatic Elevations (ISAE) automatically extracts digital terrain model (DTM) elevation points from digital aerial and satellite stereo images. The software's hierarchical image data structures and image processing methods provide a high degree of automation and accuracy. ISAE yields extremely reliable DTM points in an efficient manner due to high redundancy. This algorithm also reduces collection time by defining collection areas and skipping excluded areas. ISAE offers a robust set of features that helps increase your overall productivity [14].

ISAE produces higher-quality DTMs by combining robust digital image matching algorithms, automatic blunder detection, and a robust least squares using finite element interpolation. This method can extract DTMs from digital stereo images. ISAE is fully integrated with Image Pipe, Intergraph's image processing and display foundation. Using ISAE, you can collect DTMs from images that range between 8 bits and 16 bits in radiometric resolution. ISAE is completely multi-threaded; it is the only product of its class that is a true Symmetric Multi-Processor (SMP) application [13].As show in fig. 8.

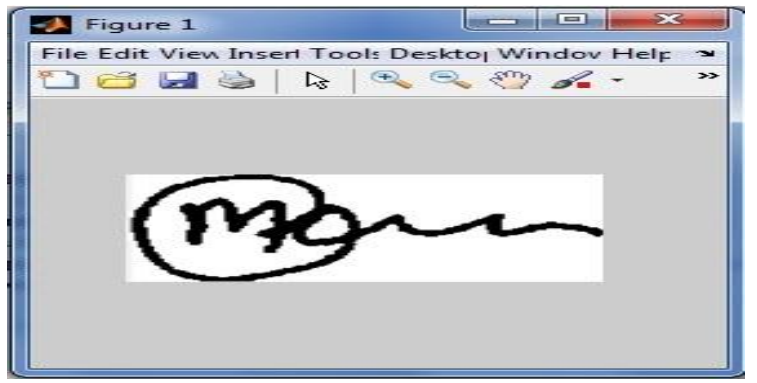

Fig.8 Image Segmentation and Cropping

\section{Edge Detection}

Edge detection is one of the most commonly used operations in image analysis. An edge is defined by a discontinuity in gray level values. In other words, an edge is the boundary between an object and the background. The shape of edges in images depends on many parameters: [12]. In this work we are using sobel edge detection. The 'Sobel' operator performs a 2-D spatial gradient measurement on an image and so emphasizes regions of high spatial frequency that correspond to edges. Typically it is used to find the approximate absolute gradient magnitude at each point in an input grayscale as show in fig. 9.

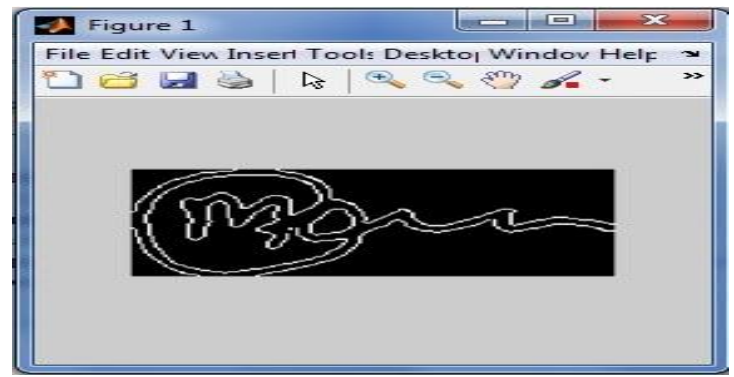

Fig.9 Edge Detection

\section{Result and Analysis}

\subsection{EXPERIMENTAL SETUP}

The proposed Extraction of signature method is tested on 50 images documents. These are selected randomly and without repetition from own database. To evaluate the efficiency of this method, human expert examines the results of the extraction of signature method from these random image documents shows a number of successful extractions of signature from Image document achieved by this method.

To evaluate the efficiency of this method human experts examine the result from these random images and show that the performance is satisfactory as show in fig. 10 .

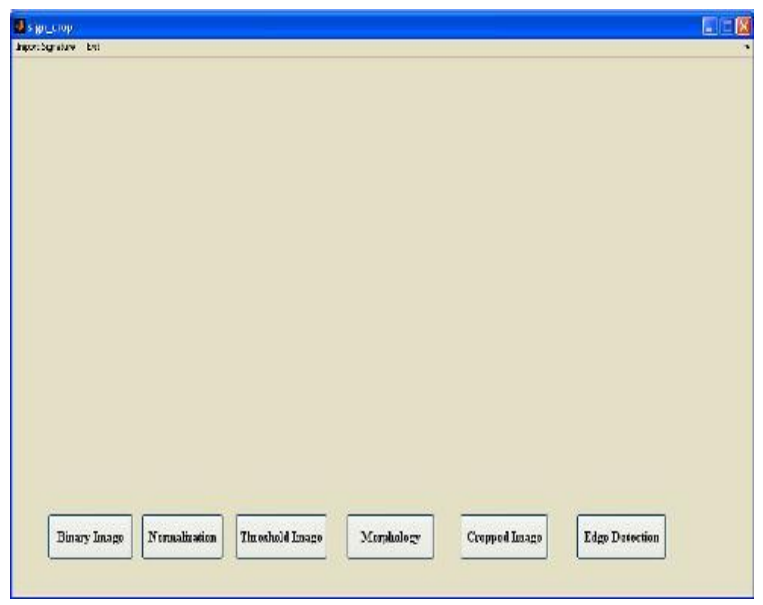

Fig.10 Graphical User Interface

\subsection{RESULTS}
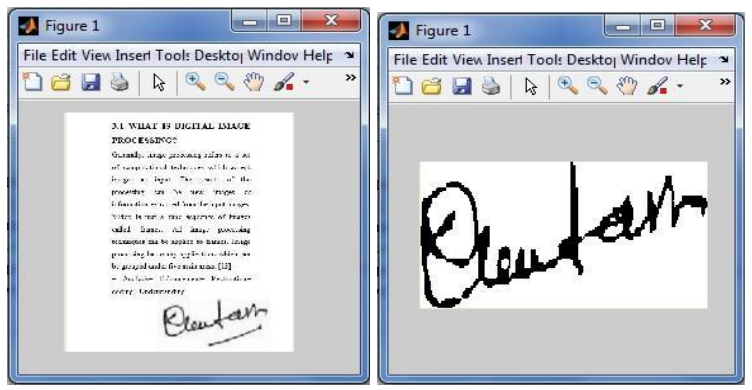

Fig.11 (a)Document Image Fig.11 (b) Result Image 

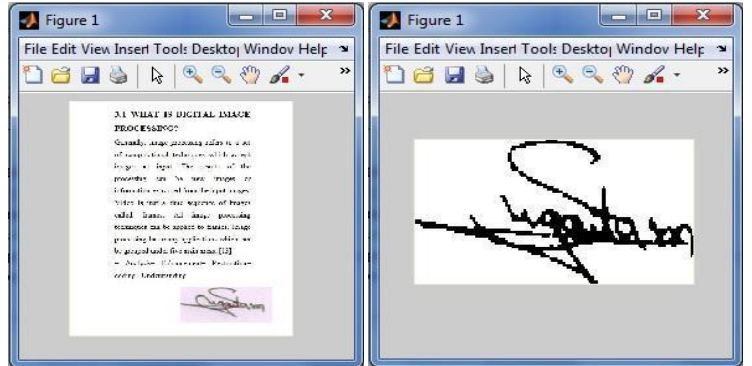

Fig.11(c) Document Image Fig.11 (d) Result Image
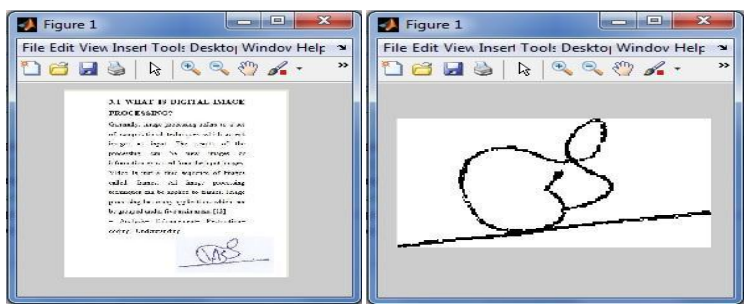

Fig.11 (e) Document Image Fig.11 (f) Result Image
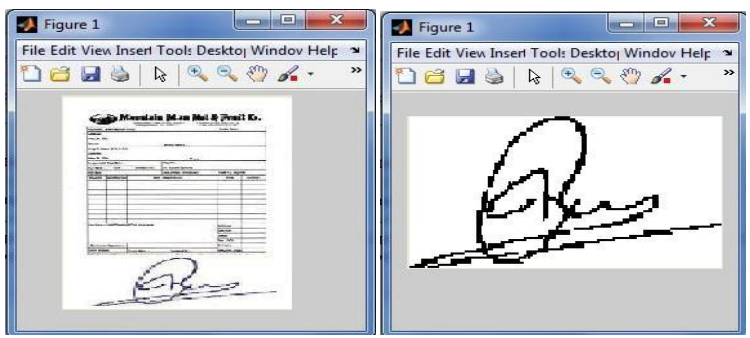

Fig.11 (g) Order form

Fig.11 (h) Result Image
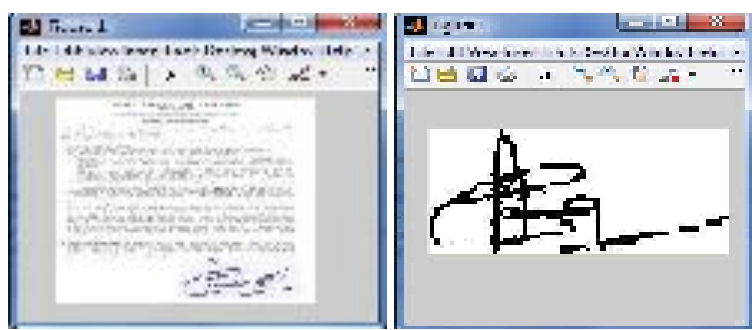

Fig.11 (i) Advertisement Form

Fig.11 (j) Result Image

\subsection{ANALYSIS}

The extraction rates of signatures from different types of documents are summarized in Table 1.

This scheme is applied on image document Database for extracted signature and the results of this processing are depicted Table 2. By using this method we get only signature. That signature can be used for security purpose. This signature can take less processing time.

\begin{tabular}{|c|c|c|}
\hline $\begin{array}{c}\text { Type of } \\
\text { Document }\end{array}$ & $\begin{array}{c}\text { Number of } \\
\text { Document }\end{array}$ & Correct Extraction (\%) \\
\hline Order form & 35 & 97.10 \\
\hline Advertisement & 40 & 98.20 \\
\hline $\begin{array}{c}\text { Document } \\
\text { Image }\end{array}$ & 35 & 100 \\
\hline
\end{tabular}

Table.1 Overall Result

\begin{tabular}{|c|c|}
\hline Processing & Processing Time (Sec) \\
\hline Binarization & 0.0902 \\
\hline Normalization & 0.0876 \\
\hline Adaptive Thresholding & 0.0742 \\
\hline Morphology & 0.0550 \\
\hline Image Segmentation & 3.1848 \\
\hline $\begin{array}{c}\text { Sobel Edge Detection } \\
\text { Total Processing Time }\end{array}$ & 0.0666 \\
\hline
\end{tabular}

Table.2 processing Time

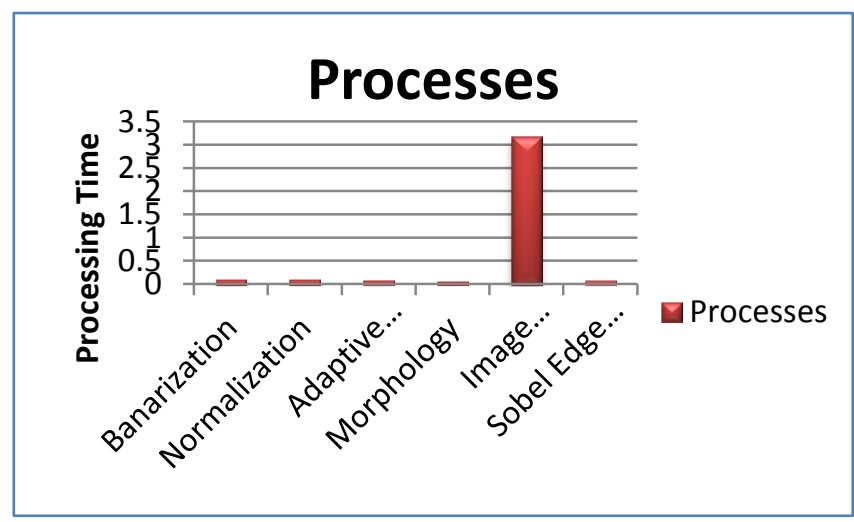

Fig. 12 Processing Time of Different Processes 


\section{CONCLUSIONS}

In This paper we proposed a new method for extract signature from image document, where the given approach shows less processing time of signature object detection and segmentation, the performance of this approach are shown through its speed time, and keeping the content information of the signature object without losing of any pixel of image. This method improves the performance of security system based on signature images as well as provides the region of interest of the used image for the biometric system. This method also reduces the time complexity associated with signature detection.

\section{FUTURE WORK}

This paper is based on image segmentation and Extraction method. This method extract signature from the image document, but if we have a word document then this method can't work. This work can be increased for word documents. This method use Image Station Automatic Elevations (ISAE) for image segmentation and automating cropping. In future, the image segmentation and cropping can be performed by other methods. Here we are using Adaptive thresholding method we will also apply other method and analyze the performance.

\section{REFERENCES}

[1] Fang, B. "Off-line signature verification with generated training samples" in IEEEProc.Vis. Image and Signal Processing. 2002.

[2] Plamondon, R. and G. Lorette, "Automatic signature verification and writer identification: The state of the art. Pattern Recognition" 22(2): p. 107-131. 1989

[3] Abuhaiba, I.S., "Offline Signature Verification Using Graph Matching”. Turk. J. Elec. Engin,. 15(1): p. 89-104. 2007

[4] Kővári, B. "Extraction of Dynamic Features for Off-line Signature Analysis". in Automation and Applied Computer Science Workshop (AACS). 2007.

[5] Santosh, K. and N. Cholwich, "A Comprehensive Survey on On-Lie Handwriting Recognition Technology and its Real Application to the Nepalese Natural Handwriting". Kathmandu University Journal of Science, Engineering and Technology. 5(1): p. 31-55. 2009
[6] Fierrez-Aguilar, J., Alonso-Hermira, N., MorenoMarquez, G., Ortega-Garcia, J., "An off-line signature verification system based on fusion of local and global information". Proc. of BIOAW, Springer LNCS-3087, p. 295-306. 2004

[7] Jason Forshaw, Ryan Volz, "Region of Interest Identification in Breast MRI Images", Applied Computer Science Workshop (AACS). 2005.

[8] Shih-Yin Ooi, Andrew Beng-Jin Teoh, Thian-Song Ong "Offline Signature Verification through Biometric Strengthening”, 1-4244-1300-1/07 IEEE.2007

[9] Miguel A. Ferrer, Jesu's B. Alonso, Carlos M. Travieso "Offline Geometric Parameters for Automatic Signature Verification Using Fixed-Point Arithmetic", IEEE Transactions On Pattern Analysis And Machine Intelligence, VOL. 27, NO. 6, JUNE 2005

[10] Derek Bradley, Gerhard Roth, “Adaptive Thresholding Using the Integral Image", Currently at The University of British Columbia.2006

[11] MamtaJuneja ,Parvinder Singh Sandhu, "Performance Evaluation of Edge Detection Techniques for Images in Spatial Domain", International Journal of Computer Theory and Engineering, Vol. 1, No.1793-8201. 5, December, 2009

[12] John C. Russ, "The Image Processing Handbook", 3rd edition CRC Press 1999

[13] Xiaoqing Liu and JagathSamarabandu, "An Edge-based text region extraction algorithm for Indoor mobile robot navigation" Proceedings of the IEEE, July 2005.

[14] www.intergraph.com and www. hexagon.com.

[15] Gonzalez, R.C., R.E. Woods, and S.L. Eddins, "Digital Image Processing USING MATLAB". : Pearson Prentice Hall .2004

[16] Vamsi Krishna Madasu, Mohd.HafizuddinMohd. Yusof, M. Hanmandluß, KurtKubik Automatic Extraction of Signatures from BankCheques and other Documents. 2003 